\title{
Review article - MCP-I: a potential target for diabetic microvascular complications?
}

\begin{abstract}
MCP-1 is a potent chemokine with the ability to mobilize and stimulate leucocytes, especially monocytes and macrophages. It is increasingly recognized as an important player in the inflammatory process that is diabetic nephropathy. In this article, we describe its role in inducing renal injury by outlining key studies in animal models and clinical studies of diabetic nephropathy, its association with diabetic retinopathy, as well as its potential use as a prognostic biomarker and as a therapeutic target in the clinical setting.
\end{abstract}

Volume 5 Issue 3 - 2017

Hsiu Lye Yap
Imperial College Renal and Transplant Centre, UK

Correspondence: Hsiu Lye Yap, Imperial College Renal and Transplant Centre, 5th Floor Commonwealth Building, Hammersmith Hospital campus, Du Cane Road, London WI2 ONN, UK, Tel 07727232 254, Email hsiulye@gmail.com

Received: August 19,2017 | Published: September 19, 2017

\section{Introduction}

Monocyte Chemoattractant Protein-1 (MCP-1) was first identified as a chemoattractant cytokine by Matsushima in $1989,{ }^{1}$ using conditioned media from a human myelomonocytic cell line. DNA cloning and sequencing of MCP-1 was completed that same year. ${ }^{2}$ Due to its domain structure of 4 cysteine residues linked by disulphide bridges, MCP-1 is also known as Chemokine (C-C motif) ligand 2 (CCL2). It was observed to strongly attract and activate monocytes/ macrophages. Apart from macrophages, MCP-1 also synchronises recruitment and infiltration of memory $\mathrm{T}$ cells $\mathrm{s}^{3}$ and natural killer cells ${ }^{4}$ to sites of inflammation. These leucocytes have been found to release inflammatory mediators such as interleukins IL-1 and IL-6, and superoxide, ${ }^{5,6}$ which contribute to the inflammatory cascade. When MCP-1 binds to its receptor CCR2, this directly causes production of fibronectin and extracellular matrix deposition in human mesangial cells. $^{7}$ Furthermore, CCR2 is also expressed on podocytes ${ }^{8}$ and renal tubular epithelial cells. ${ }^{9}$ Since the discovery of MCP-1, there have been a multitude of studies detailing its over expression and upregulation in various diseases. This review paper will particularly focus on the role of MCP-1 in diabetic nephropathy and retinopathy, before concluding on therapies targeting MCP-1.

\section{MCP-I and hyperglycaemia}

Ihm. ${ }^{10}$ first cultured human mesangial cells in high glucose media and found that these conditions directly stimulated MCP-1 expression, compared to normal glucose media. A hyperglycaemic state sets off a chain of metabolic events within the cell, leading to the creation of advanced glycation end products (AGEs) and reactive oxygen species (ROS). ${ }^{11}$ Yamagishi ${ }^{12}$ then demonstrated that AGEs increase MCP-1 and vascular endothelial growth factor (VEGF) secretion in human kidney mesangial cells, leading to glomerular hyperfiltration, which is a recognized feature of early diabetic nephropathy (Figure 1).

\section{MCP-I in diabetic nephropathy}

The pathogenesis of diabetic nephropathy is characterized by deposition of extracellular matrix, glomerular basement membrane thickening, and glomerulosclerosis. ${ }^{13}$ In 2000, Wada ${ }^{14}$ \& Banba ${ }^{15}$ found that urinary MCP-1 levels were significantly elevated in patients with diabetic nephropathy compared to healthy controls. Furthermore, urinary MCP-1 levels positively correlated with the number of interstitial macrophages and MCP-1 positive cells in kidney biopsy tissue, degree of severity of tubulointerstitial lesions ${ }^{14}$ as well as extent of albuminuria. ${ }^{15}$ It was therefore postulated that MCP-1 had a key role in inflammatory renal diseases including diabetic nephropathy, and that this was potentially mediated by macrophages.

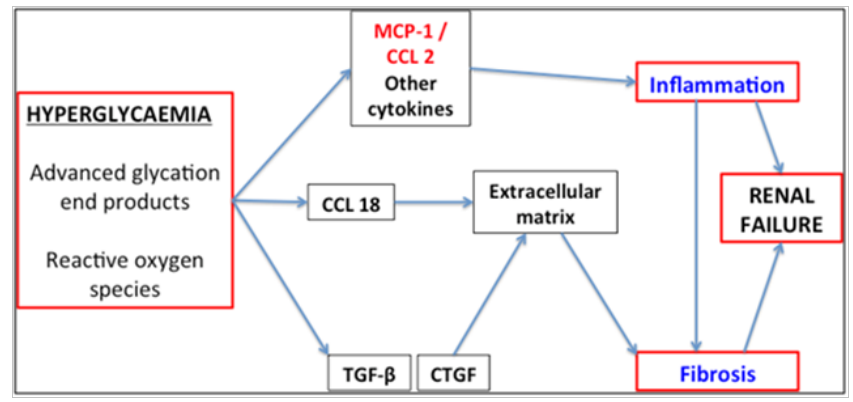

Figure I Pathogenesis of diabetic nephropathy. Hyperglycaemia results in the production of advanced glycation end products and reactive oxygen species, leading to cell signaling and release of MCP-I,TGF- $\beta$ and CTGF.These cytokines have key roles in the inflammatory and fibrotic processes that are part of diabetic nephropathy.

CCL2: CC chemokine ligand 2; TGF- $\beta$ : Transforming Growth Factor Beta; CTGF: Connective Tissue Growth Factor.

Subsequent studies in diabetic mouse models confirmed that renal injury and progression of nephropathy is mediated by macrophages. ${ }^{16}$ MCP-1 knockout mice with streptozotocin induced diabetic nephropathy had reduced macrophage recruitment and activation in the kidneys and were protected from glomerular damage, ${ }^{17}$ thus supporting the data that suggested that MCP-1 facilitates this inflammatory process. Echoing animal studies, there is a strong relationship between the amount of glomerular and interstitial macrophage accumulation in patients with diabetic nephropathy and the rate of progression of kidney failure, as measured by serum creatinine, degree of proteinuria and interstitial fibrosis..$^{18}$ Our laboratory has also shown that MCP1 release in glomerular mesangial cells is augmented by activation of the P2X7 receptor, ${ }^{19}$ a purine ATP-gated receptor that regulates cell signaling and production of inflammatory cytokines. Increased expression of $\mathrm{P} 2 \mathrm{X} 7$ receptors was detected in the renal biopsies of patients with diabetic nephropathy. The amount of glomerular macrophage infiltration and type IV collagen deposition were reduced in an experimental model of diabetic nephropathy in P2X7 receptor knockout mice, in comparison with that in wild type mice. 
Administration of a $\mathrm{P} 2 \mathrm{X} 7$ receptor antagonist reduced the number of tubulointerstitial macrophages ${ }^{19}$ and attenuated the inflammatory response in a rat model of diabetic nephropathy.

Urinary MCP-1 was initially highlighted as a potential prognostic marker for progression of diabetic renal disease in a prospective observational study where 40 patients were followed over 6years. ${ }^{20}$ It had the most predictive merit in patients with macroalbuminuria, and levels at time zero foretold the rate of decline in eGFR better than the conventionally used urine protein/creatinine ratio. Subsequently, a larger cohort of 190 patients from the ACCORD study with persistent deterioration in eGFR over 5years were matched to 190 controls with minimal decline in eGFR over the same period. ${ }^{21}$ Patients were matched for baseline demographics, medications, glycatedhaemoglobin $(\mathrm{HbA} 1 \mathrm{c})$, blood pressure, eGFR- and urine albumin-creatinine ratio. The urinary MCP-1 to creatinine ratio levels at baseline and at 24 months were compellingly linked to continued decline in eGFR, irrespective of degree of albuminuria.

\section{MCP-I in diabetic retinopathy}

MCP-1 levels in vitreous humor are significantly elevated in patients with proliferative diabetic retinopathy, and correlates with extent of activity ${ }^{22}$ and clinical staging of retinopathy. ${ }^{23}$ Another report observed a strong association between MCP-1, IL-6 and IL- 8 in patients with vitreoretinal diseases (including proliferative diabetic retinopathy and macular oedema), implying a common inflammatory pathway. ${ }^{24}$ Interestingly, our study describing urinary MCP-1's prognostic role in progression of diabetic nephropathy also reported a fourfold increase in urinary MCP-1/creatinine ratio in patients who had diabetic retinopathy. ${ }^{20}$ Therefore, urinary MCP-1 levels may predict the advent of diabetic retinopathy.

\section{MCP-I as a therapeutic target}

Currently, the mainstays of treatment for diabetic nephropathy are anti-hypertensive (including renin-angiotensin blockade) and hypoglycaemic agents. Despite these interventions, a proportion of patients can still experience progressive disease. Based on the laboratory studies, it would be interesting to investigate whether inhibiting synthesis or blocking downstream action of MCP1 improves the outcome of patients with diabetic nephropathy. Immunomodulatory therapy shows good promise to halt or delay an almost inevitable slide to end stage renal failure. The thiazolidinedione Rosiglitazone decreased MCP-1 production and recruitment of monocytes/macrophages in human mesangial cells after mechanical stretching and exposure to high glucose media. ${ }^{25}$ Specific MCP-1 blockade has also been studied in pre-clinical and clinical settings. MCP-1 receptor antagonists used in murine models have been shown to reduce mesangial matrix deposition and macrophage driven glomerulosclerosis. ${ }^{26}$ In a clinical trial, a novel selective MCP-1 receptor antagonist CCX140-B was given in addition to standard care in a randomized, double-blind study. ${ }^{27}$ Patients were randomized to placebo, $5 \mathrm{mg}$, or $10 \mathrm{mg}$ of CCX140-B daily. Reduction in albuminuria was greatest in patients receiving low dose CCX140-B, indicating MCP-1 inhibition on top of ACE inhibitors or ARBs conferred further renoprotection in diabetic nephropathy. Because of the unexpected dose ranging effect of this study, further understanding of the pharmacology of MCP-1 receptor antagonistsis still needed. The effect of MCP-1 receptor antagonists in diabetic retinopathy remains to be investigated., Proof-of principle clinical studies of $\mathrm{P} 2 \mathrm{X} 7$ receptor inhibition on renal MCP-1 production and pathogenesis of diabetic nephropathy and obesity related renal disease remain to be studied.

\section{Conclusion}

The pathogenesis of diabetic nephropathy is that of ongoing inflammation and fibrosis. Both experimental models and human research studies into MCP-1 have clarified its position in accelerating the inflammatory cascade, its relationship to the severity of renal lesions, and its use as a prognostic marker. More excitingly, new treatments for diabetic nephropathy targeting MCP-1 are producing results that are consistent with better outcomes compared to standard treatment, and will be welcome additions to our arsenal of weapons against this debilitating disease.

\section{Acknowledgements}

This work is supported by the Diamond Fund from Imperial College Healthcare Charity and the National Institute for Health Research (NIHR) Biomedical Research Centre based at Imperial College Healthcare NHS Trust. FWKT is supported by Ken and Mary Minton Chair of Renal Medicine.

\section{Conflicts of interest}

Prof FWKT has received research project grants from AstraZeneca Limited, Baxter Biosciences, Boehringer Ingelheim, and MedImmune, and has consultancy agreements with Rigel Pharmaceuticals, Novartis and Baxter Biosciences.

\section{Funding}

None.

\section{References}

1. Matsushima K, Larsen CG, DuBois GC, et al. Purification and characterization of a novel monocyte chemotactic and activating factor produced by a human myelomonocytic cell line. J Exp Med. 1989;169(4):1485-1490.

2. Yoshimura T, YuhkiN, Moore SK, et al. Human monocyte chemoattractant protein-1 (MCP-1). Full length cDNA cloning, expression in mitogenstimulated blood mononuclear leukocytes, and sequence similarity to mouse competence gene JE. FEBS Lett. 1989;244(2):487-493.

3. Carr MW, Roth SJ, Luther E, et al. Monocyte chemoattractant protein 1 acts as a T-lymphocyte chemoattractant. Proc Natl Acad Sci USA. 1994;91(9):3652-3656.

4. Maghazachi AA, al-Aoukaty A, Schall TJ. C-C chemokines induce the chemotaxis of NK and IL-2-activated NK cells. Role for G proteins. $J$ Immunol. 1994;153(11):4969-4977.

5. Jiang Y, Beller DI, Frendl G, et al. Monocyte chemoattractant protein-1 regulates adhesion molecule expression and cytokine production in human monocytes. J Immunol. 1992;148(8):2423-2428.

6. Zachariae $\mathrm{CO}$, Anderson AO, Thompson HL, et al. Properties of monocyte chemotactic and activating factor (MCAF) purified from a human fibrosarcoma cell line. J Exp Med. 1990;171(6):2177-2182.

7. Giunti S, Tesch GH, Pinach S, et al. Monocyte chemoattractant protein-1 has prosclerotic effects both in a mouse model of experimental diabetes and in vitro in human mesangial cells. Diabetologia. 2008;51(1):198 207.

8. Lee EY, Chung $\mathrm{CH}$, Khoury CC, et al. The monocyte chemoattractan protein-1/CCR2 loop, inducible by TGF-beta, increases podocyte motility and albumin permeability. Am J Physiol Renal Physiol. 2009;297(1):F85-F94. 
9. Viedt C, Dechend R, Fei J, et al. MCP-1 induces inflammatory activation of human tubular epithelial cells:involvement of the transcription factors, nuclear factor -kappa B and activating protein-1. J Am Soc Nephrol. 2002;13(6):1534-1547.

10. Ihm CG, Park JK, Hong SP, et al. A high glucose concentration stimulates the expression of monocyte chemotactic peptide 1 in human mesangial cells. Nephron. 1998;79(1):33-37.

11. Brownlee M, Cerami A, Vlassara H. Advanced glycosylation end products in tissue and the biochemical basis of diabetic complications. $N$ Engl J Med. 1988;318(20):1315-1321.

12. Yamagishi S, Inagaki Y, Okamoto $\mathrm{T}$, et al. Advanced glycation end product-induced apoptosis and overexpression of vascular endothelial growth factor and monocyte chemoattractant protein-1 in humancultured mesangial cells. J Biol Chem. 2002;277(23):20309-20315.

13. Cooper ME. Pathogenesis, prevention, and treatment of diabetic nephropathy. Lancet. 1998;352(9123):213-219.

14. Wada T, Furuichi K, Sakai N, et al. Up-regulation of monocyte chemoattractant protein-1 in tubulointerstitial lesions of human diabetic nephropathy. Kidney Int. 2000;58(4):1492-1499.

15. Banba N, Nakamura T, Matsumura M, et al. Possible relationship of monocyte chemoattractant protein-1 with diabetic nephropathy. Kidney Int. 2000;58(2):684-690.

16. Chow F, Ozols E, Nikolic-Paterson DJ, et al. Macrophages in mouse type 2 diabetic nephropathy :correlation with diabetic state and progressive renal injury. Kidney Int. 2004;65(1):116-128.

17. Chow F, Nikolic-Paterson DJ, Ozols E, et al. Monocyte chemoattractant protein-1 promotes the development of diabetic renal injury in streptozotocin-treated mice. Kidney Int. 2006;69(1):73-80.

18. Nguyen D, Ping F, Mu W, et al. Macrophage accumulation in human progressive diabetic nephropathy. Nephrology (Carlton). 2006;11(3):226-231.
19. Menzies RI, Booth JWR, Mullins JJ, et al. Hyperglycemia-induced renal P2X7 receptor activation enhances diabetes-related injury. EBioMedicine. 2017;19:73-83.

20. Tam FWK, Riser BL, Meeran K, et al. Urinary monocyte chemoattractant protein-1 (MCP-1) and connective tissue growth factor (CCN2) as prognostic markers for progression of diabetic nephropathy. Cytokine. 2009;47(1):37-42.

21. Nadkarni GN, Rao V, Ismail-Beigi F, et al. Association of urinary biomarkers of inflammation, injury, and fibrosis with renal function decline:the ACCORD trial. Clin J Am Soc Nephrol. 2016;11(8):13431352.

22. Abu El-Asrar AM, Struyf S, Kangave D, et al. Chemokines in proliferative diabetic retinopathy and proliferative vitreoretinopathy. Eur Cytokine Netw. 2006;17(3):155-165.

23. Tashimo A, Mitamura Y, Nagai S, et al. Aqueous levels of macrophage migration inhibitory factor and monocyte chemotactic protein-1 in patients with diabetic retinopathy. Diabet Med. 2004;21(12):1292-1297.

24. Yoshimura T, Sonoda KH, Sugahara M, et al. Comprehensive analysis of inflammatory immune mediators in vitreoretinal diseases. PLoS One. 2009;4(12):e8158.

25. Gruden G, Setti G, Hayward A, et al. Mechanical stretch induces monocyte chemoattractant activity via an NF-KB-dependent monocyte chemoattractant protein-1-mediated pathway in human mesangial cells:inhibition by rosiglitazone. J Am Soc Nephrol. 2005;16(3):688696.

26. Kanamori H, Matsubara T, Mima A, et al. Inhibition of MCP-1/CCR2 pathway ameliorates the development of diabetic nephropathy. Biochem Biophys Res Commun. 2007;360(4):772-777.

27. de Zeeuw D, Bekker P, Henkel E, et al. The effect of CCR2 inhibitor CCX140-B on residual albuminuria in patients with type 2 diabetes and nephropathy:a randomised trial. Lancet Diabetes Endocrino. 2015;3(9):687-696. 